\title{
HYBRID REPRESENTATION OF DIGITAL MOCKUP FOR HERITAGE BUILDINGS MANAGEMENT
}

\author{
G. Nicolas ${ }^{\mathrm{a}}$, J. Landrieu ${ }^{\mathrm{a}}$, Y. Nugraha ${ }^{\mathrm{a}}$, C. Père ${ }^{\mathrm{a}}$ \\ ${ }^{a}$ Laboratoire Electronique, Informatique et Image (LE2I) - Université de Bourgogne - Arts et Métiers ParisTech - \\ CNRS : UMR 6306.
}

KEY WORDS: Digital mock up, BIM, Mixed reality, Old buildings, 3D-Layers

\begin{abstract}
:
This article deals with the implementation of tool allowing the portability of the digital mock-up for architectural projects on the building renovation place and the use of representation layers giving functions adapted to the different workers open to work in this place. Our test case is applied to renovation works on old windows in an ancient abbey where it is necessary to improve the thermal efficiency.
\end{abstract}

\section{INTRODUCTION}

In the building construction world, the BIM (Building Information Model) tends to be imperative. This kind of mock-ups enriches with lots of information all the element of the buildings. This allows industrials to capitalize knowledge according to three dimensions: space, time and fields of expertise. The biggest interest of such a mock-up is gathering all professional of construction together on the same representation of the building. This virtual representation of the future building is designed with CAD software in an orthonormal space. The real construction is tending to be as close as possible to the virtual ideal representation of the building.

In the case of ancient building renovation, the context is different because the building already exists. The traditional techniques did not always allow making perfect work geometrically and the building has been deteriorated by the time. In these heritage buildings, structures are not geometrically simple; moreover walls are neither planes nor perfectly vertical and angles are not straight. This is not compatible with the norms of BIM modelling. (Hichri, 2013) Some constraints of conservation also require preserving many historic elements. The current research in the field of automatic shape recognition is not matured enough and still many operations are made manually which is complex and time consuming. This explains why BIM is delaying in being used for old buildings.

\section{STATE OF THE ART}

\subsection{Modelling}

Nowadays, the ways of capturing the geometry of a building are being developed and they become more precise, easier to use and easier of access. The laser scanner devices can also acquire thousands of points of the building each second. (Boehler, 2002) They are also a really interesting way to create faithful digital representations of buildings as a point cloud. Some devices are also able to put colours on this cloud or capture information about the materials.
A second way of capture is photogrammetry. Only some pictures are necessary to create the 3D-model however the coordinates are characteristic of the mock-up, not of the world. (De Luca, 2006)(Deveau, 2006)(Cerovsek, 2011)

\subsection{Mockup enrichment}

Even if the mock-up created by laser scanners or photogrammetry algorithms gives lots of information about dimensions and visualizations, it doesn't contains any semantic information, like BIM models do. Many studies are working on algorithms for creating rich models from the conversion of a point cloud. This conversion process from a point cloud to a BIM mock-up is called "scan-to-BIM". The process described by (Deschaud, 2010) is interesting as a first process to apply on the point cloud.

Then, the study deals with associating surfaces and simple solids for each area of the point cloud. These elements represent walls, floors, ceilings, doors... and contain lots of metadata like materials and structural information. Algorithms are studied to automate this process which needs an important manual and time-consuming intervention of competent people at the present time. (Tang, 2010) (Xiong, 2013) The other issue is that the modelling could be different from one user to another because of the manual operations: automating the process is the solution for this problem.

Finally, scan-to-BIM offers an interesting, rich model of buildings, but this is still an approximate representation of the reality like any mock-up. Moreover, the process of retroconception is really time-consuming.

Despite these remarks, the digital mock-up is the right tool to define all the information of the building all along its life cycle. The mock-up is an approximate representation and is the only way to store BIM/IFC data. Thus, this is a complementary model of the point cloud that only represents the perfect geometry of the building. Each of the different representations contains many interesting and complementary data. (Busarayat, 2010) We have to put some links between all these models to connect all the data together. 


\subsection{Information representation}

The numerous items of information in the BIM representation of a building forces us to organize smartly all the data. The BIM mock-up is a theoretical model of the building "as-defined" and is a reference. The "as-build" representation of the building is described by the point cloud. The use of 3D environment allows us to use multiple metaphors of representations to differentiate each type of information. Also we want to have multiple representations' modes corresponding to all the use we have of the mock-up and the data we search. That's why we will opt for a job point of view; we will have a different representation for all the jobs working on the construction: this will be named "job views". In this idea, we will transpose the concept of layers like they are used in GIS (Brooks, 2008) in a 3D environment. They will be 3D-layers. Each layer will only allow access to the function the professional need. The concept of layer shows the easiness for user to access one information or another in the mock-up, or multiple information together.

\subsection{Actual portable applications}

We want our work to be accessible for professional on the place of work. Also we decided to develop a portable application for pad devices using Android or iOS. Today, there are some applications allowing managing building site. They give access architect's plans and schedule information but nothing deals with 3D representation.

\section{PROBLEMATIC}

The goal of our project is to create a 3D portable application adapted to all the professionals working on building that could replace their actual ways of building management, being easier to use and taking advantage of BIM methods.

We have developed the application with the game engine Unity3D which is adapted for 3D application and export on portable devices.

Finally, our goal is to show the efficiency of an access to the digital mock-up of a building for the entire professional on the construction site. (Landrieu, 2012) Then, we will have a method to improve the concept of job oriented layers giving the right tools to each professional user.

\section{TECHNICAL APPROACH}

The objective of this study was to analyse as-built configuration and to establish the relevance to handle multi-representation of the mock-up.

\subsection{Data survey}

In order to get accurate data from current configuration, the room dedicated to the use case was scanned. FARO Focus3D device was placed at the center of the area of interest. Two views were necessary to acquire the whole volume with high resolution textured points. As two sub-configurations were recorded (shutters opened or not), this step spent about forty minutes.

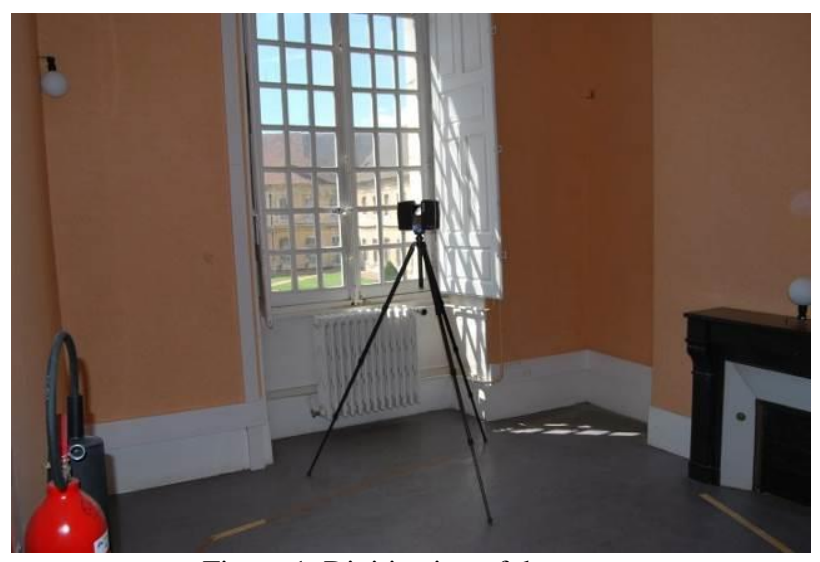

Figure 1. Digitization of the room

\subsection{Data Pre- and Post-processing}

Once the survey was finished, data could be transferred to "Scene" software. It is developed by FARO incorporation to convert laser scanner data to points clouds. In the present case two views were automatically aligned thanks to the spheres recognition. Point clouds were exported in .pts format and imported under Rapidform XOS software. This step permitted to compute meshes and to generate levels of detail. Besides point clouds were too heavy and decreased the performance of post-treatment. Thus the whole points cloud was discretized into multiple lighter point clouds which correspond to architectural parts of the room: the walls

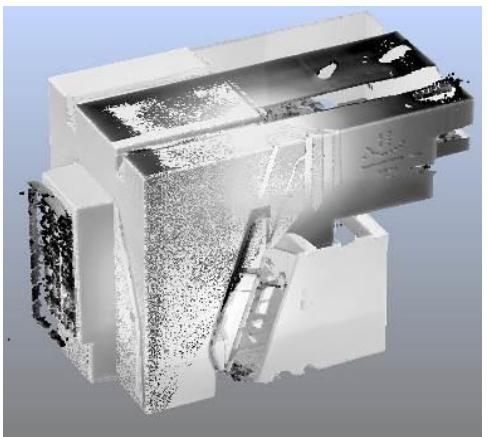

Figure 2. Example of scan data under FARO Scene

\subsection{Flatness analysis}

As for a first analysis of the current configuration, we aimed at studying the flatness of the facades. The meshes of each facade were imported into a CAD modeller in order to obtain the average plane. It was imported under CloudCompare to analyse the mesh and the theoretical flat facade. Results can be found bellow. 


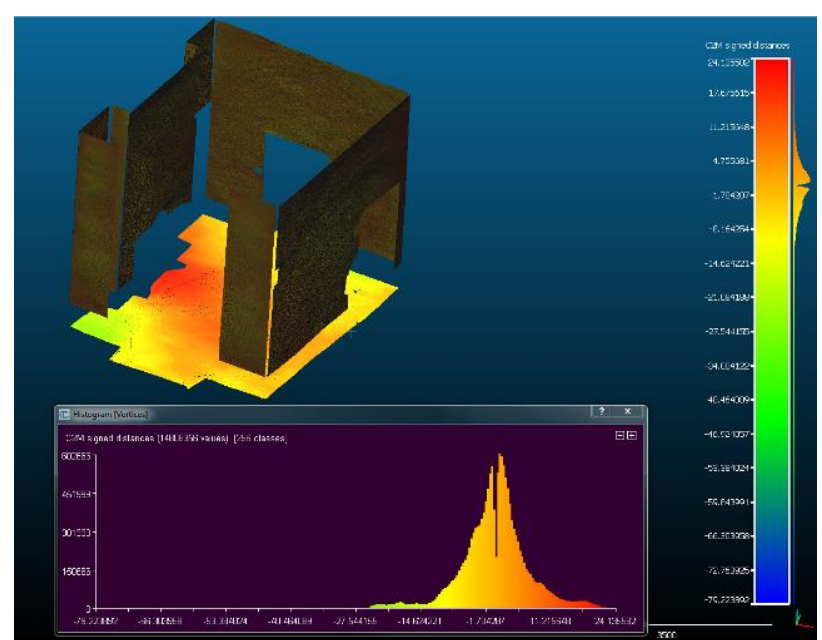

Figure 3. Re-assembled decimated meshes compared to perfect flat planes

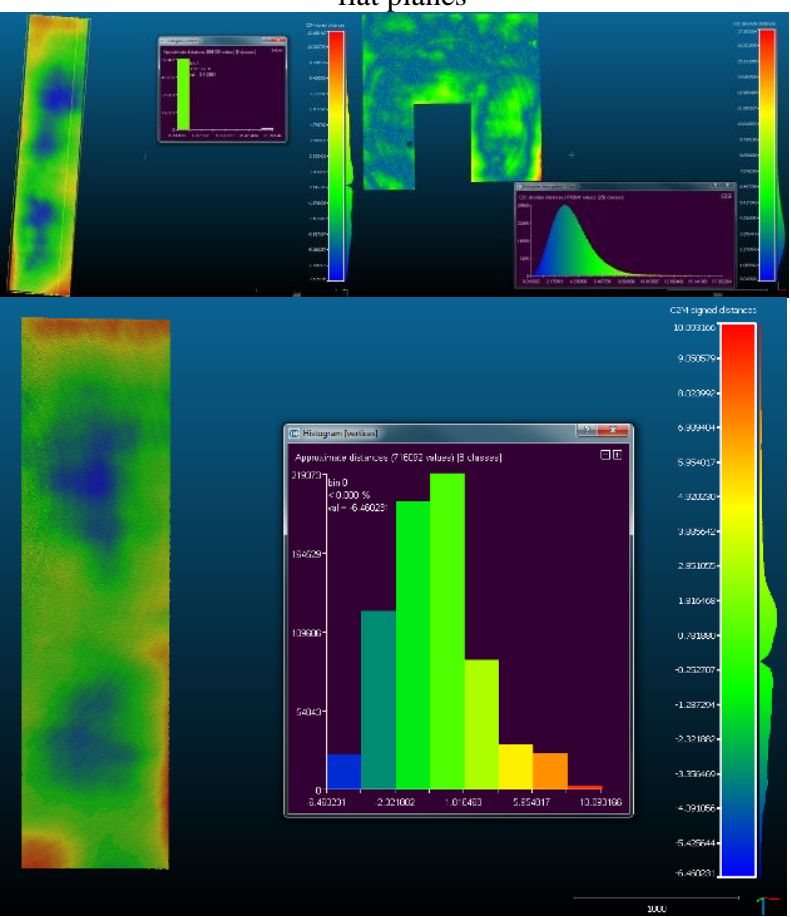

Figure 4. Re-assembled decimated meshes compared to perfect flat plane

\subsection{Verticality check.}

In addition with the previous study, we aimed at analysing the verticality of the walls. To do that, we followed the deviation between the true vertical (measured by a sensor embedded in the scanner) and the edges which result from the intersection between perfect planes. Results show differences up to 63millimeters on an average height of 3,9 meters.

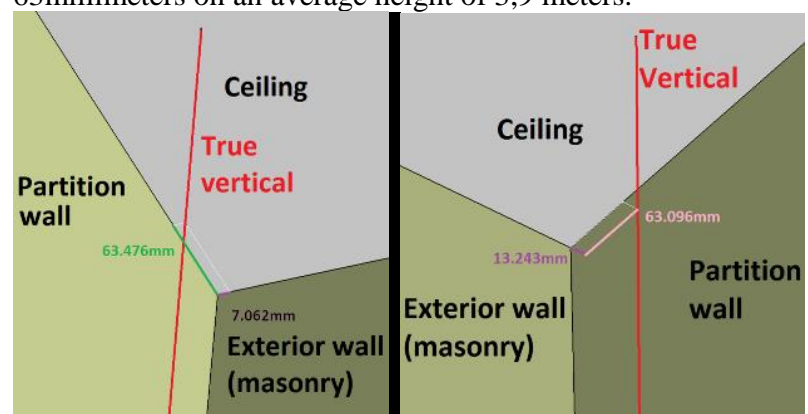

\section{APPLICATION}

The application we develop has to be adapted for any professional working on ancient building restoration: architects, project manager, workers... For a first development, we used the mock-up of an office room in the abbey of Cluny. The building is now use by the highschool Arts et Métiers Paristech. The restoration of this room concerns mainly the window and the heater.

\subsection{Scenes}

We are using 3 scenes representing all the models of the building: a point cloud representing the present state of the room is the "as-build" representation; a mock-up of actual state is the "as-defined" representation; and a mock-up representing the future state of the room like we would like it to be after restoration is the "as-planned" representation. These three models allow us to represent all the states of restoration of the building during the work.

The point cloud is created by a laser scanner and converted to a $3 \mathrm{D}$ object. It's the critical object of the application because of its high density of points. It also has to be lightened so that the mobile devices, which are not as powerful as computers, could well render it.

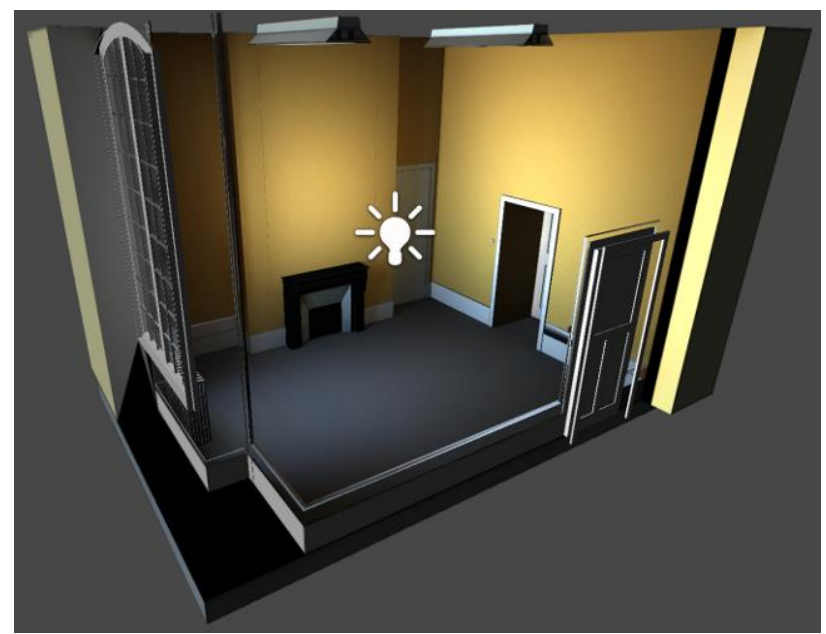

Figure 5. "as-defined" model in Unity3D

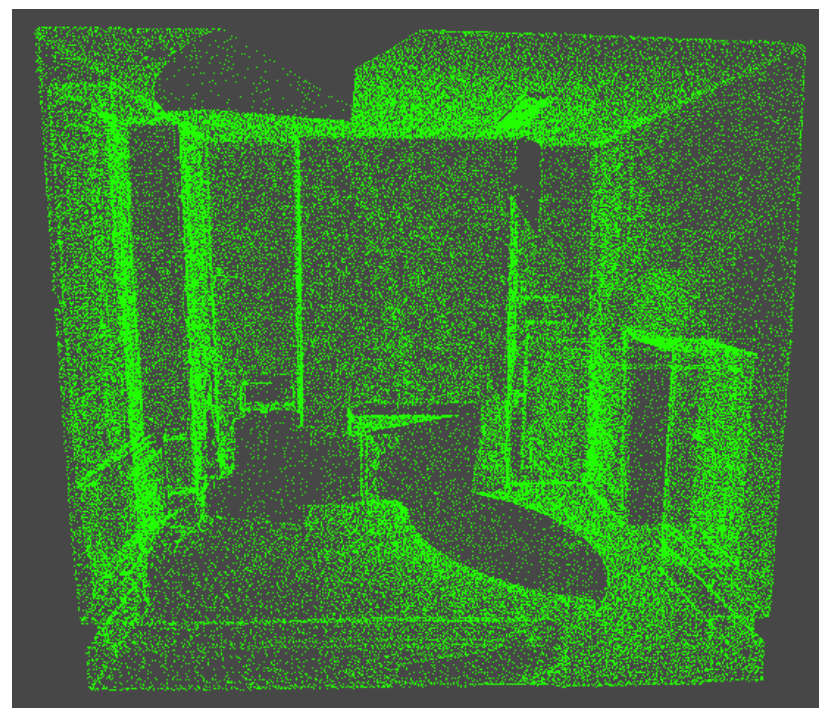

Figure 6. "as-build" model in Unity3D (point cloud) 


\subsection{Mixed reality}

To make the use of our application easier for people who are not accustomed to use such models, we decided to put him in a mixed reality. The role of the application is to react like if the user was moving into the mock-up when moving the device. This interaction is possible using gyroscope and compass of the pad. Then, when the pad is turned around, the camera in the virtual scene is turning too. Also, the view of the user of the reality around him and the screen of the device are the same place at a different moment. With this co-localisation, it is easy for people to find their way around the scene, they are free to move, the device is like a window or their eyes looking into the mock-up instead of the reality.

\subsection{Functions}

First of all, we have developed many functions useful for people working on a construction site. The list is not exhaustive of all the functions that could be imagined or will be used by professionals but represent the main ones.

\subsubsection{Measurements}

Users can take measurements into the application and they have 2 choices. Measurements can be done on the point cloud to have the exact dimension of the present room or on one of the mock-up to get the ideal dimension of an element.

They just have to point the places where they want measurements to be done and the dimension is shown on the screen.

\subsubsection{Simulated data}

With actual BIM models and computer technologies, it is possible to do multiple types of numeric simulation in any environment. Also, it can be interesting to get some of them into our application so that professional could consult it whenever they want. In that case, we've got thermic simulation data in the test scene. It shows the temperature everywhere in the scene and

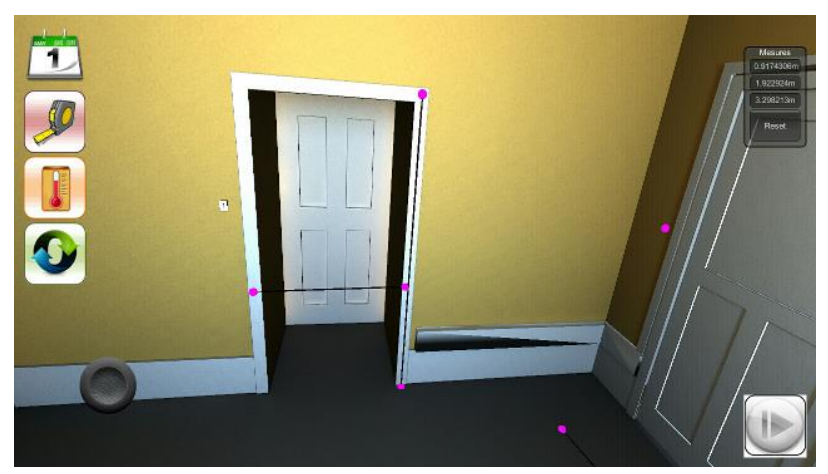

Figure 8. Taking measurements

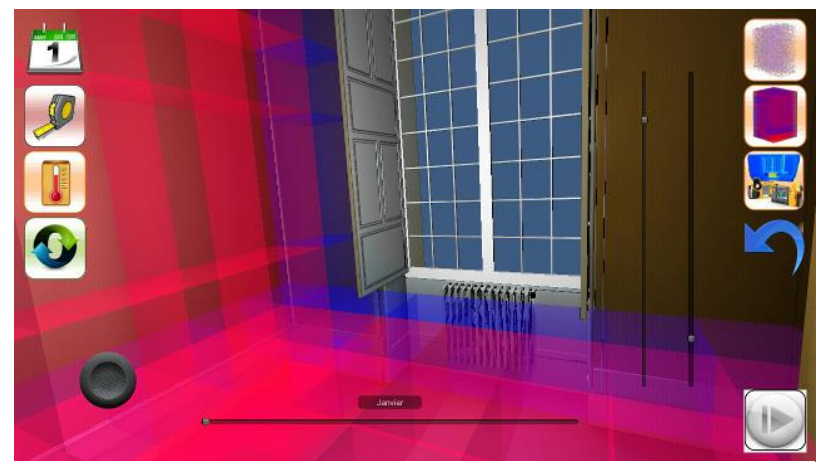

Figure 7. Visualization of simulated data every month during one year.

These data are calculated by external software and imported in the application by a text file.

\subsubsection{Accessing metadata}

Metadata are all the BIM information contained in the BIM model of the mock-up. In that case, the mock-up doesn't already contain all those data. Also, it was decided to use an external way to create this information. In this way, it will be easier for the users of the application to modify the pieces of information without using Unity3D but only a database. This was made using Excel and exporting the document as a .csv file. This type of text file can be read by Unity.

At launch of the application, all pieces of information in the data base are imported and put on every parts of the scene which correspond them. Then, when pointing at any object, the associated data are shown on the top right corner.

\subsubsection{Work schedule}

This last function shows the schedule of the building work from its state of ancient building to renovated one. It uses the two mock-ups describing the two states of the edifice and show or hide all the elements of the scene one by one according to the schedule planed. Users can see the models evolving during time; it's an easy way to locate the present state of the renovation.

\section{EXPERIMENTATIONS}

The scientific approach of our project is to show the utility of using job oriented layer for the representation of information in our portable application compared to classic methods of managing renovation work.

We will bring our experimentations into two ways

The first one will be to identify the need of each professional among the functions we develop. The second one is to propose our application on a renovation work and observe the use people have of the application and receive their impression.

\subsection{First approach}

The goal of this first part of our experimentation is to determine the content of each "job-layer" according to the needs of all professional.

Different scripts will be written for each job in each subject will have to accomplish several tasks corresponding to the current job. These scripts will show the maximum of the tasks the professionals are doing every day. Next are some of the tasks that will be controlled.

- Locating: the first task to accomplish is locating yourself in the environment. The mixed reality is efficient to

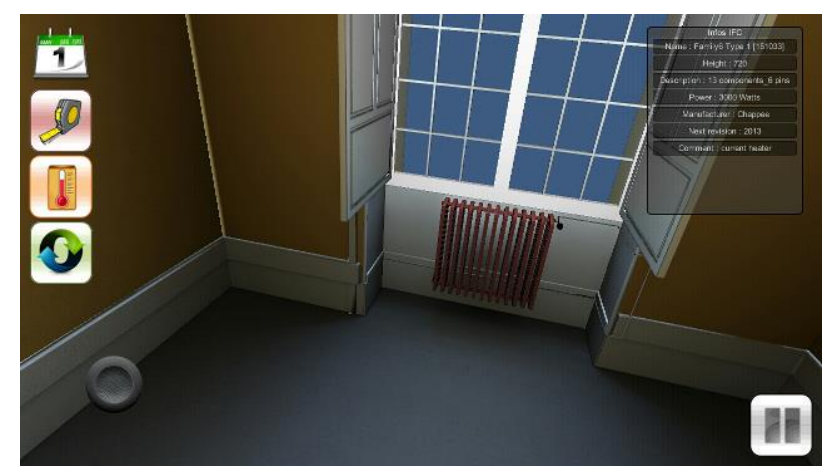

Figure 9. Show metadata 
make it easier but people who are not used to the representation of 3D mock-up could have trouble. While users don't understand how this works, they can't use anything else in the application.

- Current state: the digital mock-up can represent anyone if the state of the building between the beginning and the end of the renovation. The user will have to navigate along the chronology to match the state of the virtual representation with the reality.

- Manage work: once the current state defined, we can ask user to manage their workers. Also, we can ask them to determine the next tasks he will ask to do, like removing the window, or to order material for further tasks.

- Taking measurements: Subjects will be asked to take multiple measurements on the "as-build", "as-defined", or "as-planed" models.

- Simulated data: accessing simulated data could be interesting for some jobs. According to this, we have implemented thermic simulation which could be useful for heat engineer for example. In that case, subjects will be asked to identify important elements of the data like coldest or hottest areas of the room and area where temperature is changing the more over one year.

- Objects characteristics: concerning metadata, we will ask subject to give some pieces of information about them and compare it to the rest of the scene. Moreover we will ask them if we put enough data or if they need more.

Finally, we will ask all subjects to complete more complex tasks that will require the use of multiple functions. This will show highlight how much subjects have understand the logical approach of our application.

During this experiment, we will collect multiple piece of information observing the subjects and asking them some questions. First of all, we will have the time they needed to accomplish the tasks, the number of mistakes they made. Then we will have their impression about the easiness of use, feeling of immersion, the learnability, and the speed of use.

\subsection{Second approach}

After the first study, one parameter will allow us to create "job views" while other will help to improve the application; it's the time subjects spent using each function for each script. According to this, we will be able prioritize some layers for each job and see which one are not useful. According to this, we will create a job view for each professional only including the functions that are useful for him and not the ones he will never use.

Once the "job views" will be created, we will apply our application to a real building work and collect more information to improve the application again.

\section{PERPECTIVES}

In a near future, we are looking for an ancient building close to be renovated which could be a test for our application. According to the approach we defined above, our application could be adapted to this work by changing our test room by the models of this building. Then, we will give many pads with the application to the workers. Regularly, we will collect their feelings about the use they have of our applications. This fullscale test will give us the final result about the usefulness of the mobile application and the job layers representation.

\section{REFERENCES}

Boehler W., Heinz G. Marbs A. (2002). The potential of noncontact close range laser scanners for cultural heritage recording. The International archives of Photogrammetry, Remote Sensing and Spatial Information Sciences, Vol. 34(5/C7), pp430-436.

Brooks S. and Whalley J.L. (2008), Multilayer hybrid visualizations to support 3D GIS, Computers, Environment and Urban Systems, Vol. 32, Issue 4, pp278-292

Busayarat C. (2010). La maquette numérique comme support pour la recherche visuelle d'informations patrimoniales, mémoire de thèse de doctorat.

Cerovsek T. (2011). A review and outlook for a 'Building Information Model' (BIM): A multistandpoint framework for technological development, Advanced Engineering Informatics, Vol.25, pp224-244.

De Luca L. (2006). Relevé et représentations du patrimoine architectural - méthodes, formalismes et outils pour l'observation dimensionnée d'édifices.

De Luca L. (2006). Relevé et multi-représentations du patrimoine architectural. Méthodes, formalismes et outils pour l'observation dimensionnée d'édifices, MIAjournal (Informative Modelling for the Architectural heritage) Volume 0, Numéro 1. pp131-142.

Deschaud M. (2010). Traitements de nuages de points denses et modélisation $3 D$ d'environnements par système mobile LiDAR/Caméra.

Deveau M. (2006). Utilisation conjointe de données image et laser pour la segmentation et la modélisation $3 D$.

Hichri N., Stefani C., De Luca L. and Veron P. (2013). Review of the « as-built BIM » approaches, 3D Arch 2013 - 3D Virtual Reconstruction and Visualization of Complex Architectures (Trento, Italy, 25-26 February 2013)

Landrieu J., Nugraha Y., Merienne F., Père C. and Nicolle C. (2012). Pertinence d'une représentation $3 D$ d'un projet de rénovation $d u$ bâtiment en réalité augmentée mobile, à CONFERE 2012 (San Servolo Venise, 5-6 Juillet 2012)

Tang P., Huber D., Akinci B., Lipman R., Lytle A. (2010). Automatic reconstruction of as-built building information models from laser-scanned point clouds: A review of related techniques, Automation in Construction, Vol. 19, Issue 7, pp829-843.

Xiong X., Adan A., Akinci B., Huber D. (2013). Automatic creation of semantically rich $3 \mathrm{D}$ building models from laser scanner data, Automation in Construction, Vol. 31, pp 325-337. 